Check for updates

Cite this: RSC Adv., 2019, 9, 21323

\title{
Solidly mounted resonator sensor for biomolecule detections
}

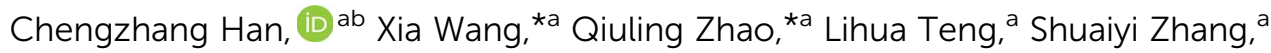 \\ Hao Lv, ${ }^{a}$ Jing Liu, ${ }^{a}$ Haoran $\mathrm{Ma}^{a}$ and Yanping Wang ${ }^{a}$
}

We report the fabrication of a solidly mounted resonator (SMR) that can also function as a sensor for biological molecules. The SMR, consisting of a Au electrode, aluminum nitride (AIN) piezoelectric thin film and Bragg acoustic reflector, was fabricated on a Si substrate by radio frequency (RF) magnetron sputtering. The Bragg acoustic reflector, made entirely of metal, has small internal stress and good heat conduction. Human immunoglobulin G ( $\mathrm{gG}$ ) antibody was immobilized on the modified (by selfassembled monolayer method) Au electrode surface of the SMR and goat anti-human IgG antigen was captured through the specificity of bond between the antibody and antigen on the electrode surface. We found a linear relationship between the resonant frequency shift and the concentration of goat antihuman IgG antigen for concentrations smaller than $0.4 \mathrm{mg} \mathrm{ml}^{-1}$ and a relatively constant frequency shift for concentrations greater than $0.5 \mathrm{mg} \mathrm{m}^{-1}$. A series of interference experiments can prove that the selectivity of the sensor is satisfactory. Our findings suggest that the SMR sensor is an attractive alternative for biomolecule detection.

Received 6th March 2019

Accepted 30th June 2019

DOI: $10.1039 / \mathrm{c} 9 \mathrm{ra01695c}$

rsc.li/rsc-advances operation frequency (5-20 MHz) due to the bulk quartz substrate thickness. Thus BAW sensors do not have adequate sensitivity for the detection of small molecules in low concentrations. ${ }^{\mathbf{1 4 , 1 5}}$ With the advancement in micro/nano fabrications, film bulk acoustic resonator (FBAR) is proposed as a typical MEMS piezoelectrical device. Importantly FBAR operating in the $\mathrm{GHz}$ range can overcome the shortcomings of BAW sensors and have better performance in mass-sensitive detections. ${ }^{\mathbf{1 6}}$ In addition to the sensitivity, FBAR has several other advantages, including small in size, room-temperature operation, and mass producible. ${ }^{17-19}$

Piezoelectric materials such as zinc oxide $(\mathrm{ZnO})^{20}$ and aluminum nitride (AlN) ${ }^{21}$ have been used in FBAR devices for various applications owing to their high acoustic velocity, better quality factor, and high electromechanical coupling coefficient. ${ }^{22-24}$ There are two types of FBARs, one with a freestanding membrane and the other solidly mounted resonator (SMR) composed of a piezoelectric thin film sandwiched between electrodes and Bragg reflector consisting of alternating high and low acoustic impedance quarter-wavelength thick dielectric or metallic layers. ${ }^{25,26}$ By comparing and studying, the SMR with the simple fabrication, good mechanical strength, excellent acoustic properties and closer to CMOS integration was fabricated as a promising candidate for biomolecule detections. ${ }^{27,28}$

In this paper, we explored the possibility of AlN film SMR as a sensor for biomolecule detections. We have fabricated SMR device, immobilized human immunoglobulin G (IgG) antibody on the SMR electrode surface modified by self-assembled monolayer (SAM) method for the detections of goat anti-human IgG antigen. ${ }^{29}$ The sensitivity and usability of sensor were evaluated for goat antihuman IgG antigen detection. In addition, the relationship between
${ }^{a}$ Optoelectronic Materials and Technologies Engineering Laboratory, Shandong, Physics Department, Qingdao University of Science and Technology, Qingdao 266042, China.E-mail: phwangxia@163.com; sdqlzhao@163.com

${ }^{b}$ College of Mechanical and Electronic Engineering, Qingdao Binhai University, Qingdao, 266555, China 
the resonant frequency shift of SMR sensor and the concentration of goat anti-human IgG antigen was also investigated.

\section{Experiment}

\subsection{Reagents and materials}

All chemicals and solvents were of reagent grade or better. 11Mercaptoundecanoic acid (11-MUA), 1-(3-dimethylaminopropyl)-3ethylcarbodiimide hydrochloride (EDC), $N$-hydroxysuccinimide (NHS) and bovine serum albumin (BSA) were purchased from Sigma-Aldrich. Human IgG, goat anti-human IgG, goat anti-mouse IgG and goat anti-rabbit IgG were purchased from Shanghai Dingguo Biotechnology Co., Ltd (Shanghai, China). The supporting electrolyte was $0.1 \mathrm{M}$ phosphate buffer solution (PBS) prepared with $\mathrm{Na}_{2} \mathrm{HPO}_{4}$ and $\mathrm{KH}_{2} \mathrm{PO}_{4}$. Doubly distilled water was used for preparing all the solutions.

\subsection{Apparatus}

The crystalline structure of the SMR was investigated by X-ray diffraction (XRD, Bruker Advanced D8) using a $\mathrm{Cu}-\mathrm{K} \alpha$ radiation $(\lambda$ $=1.54187 \AA$ ) in a $\theta-2 \theta$ scanning mode. The cross-sectional morphology of SMR was observed using a field emission-scanning electron microscope (FE-SEM, Carl Zeiss Ultra55). The sputtering was carried out by a multifunctional nano preparation system (Yuhua CS-350). Finally, the frequency response of SMR was measured by $S$-scattering parameters with a probe station (Cascade EPS 150 RF) and a network analyzer (HP 8712E). All measurements of response of the SMR were carried out in an ambient temperature kept at $25{ }^{\circ} \mathrm{C}$.

\subsection{SMR fabrication}

The procedures of the SMR fabrication were be divided into three steps: Bragg reflector deposition, AlN thin film fabrication, and top $\mathrm{Au}$ electrode deposition. Before the formal fabrication, the process parameters of AlN thin film were optimized repeatedly to obtain high $c$-axis orientational quality. ${ }^{30}$ The Bragg reflector, consisting of titanium (Ti) and tungsten (W) layers, was first deposited on p-type 3 inch $\mathrm{Si}(100)$ substrate with $1-10 \Omega \mathrm{cm}$ resistivity at $25^{\circ} \mathrm{C}$, and then a AlN thin film was deposited on the top of the Bragg reflector at $300{ }^{\circ} \mathrm{C}$. Lastly, a Au film was deposited on top of the AlN for the electrodes. The AlN thin film was obtained by a $\mathrm{RF}$ reactive magnetron sputtering system with an $\mathrm{Al}$ target in a $\mathrm{N}_{2}$ and $\mathrm{Ar}$ mixture atmosphere. Similar to the AlN, Ti, W, and Au film were also obtained by the RF magnetron sputtering system using Ti, W, and Au targets in a pure Ar atmosphere, respectively, with about a $70 \mathrm{~mm}$ target-to-substrate distance. The purities of all the targets are $99.999 \%$ and the diameters of all targets are $80 \mathrm{~mm}$. Finally, a thermal annealing process at $300{ }^{\circ} \mathrm{C}$ was also performed to relieve the stress in multilayer films to improve the performance of the SMR.

The specific sputtering parameters for different materials in our experiment are summarized in Table 1 . The fabrication process flow and schematic illustration of SMR are shown in Fig. 1.

\subsection{Biomolecules immobilization}

The biomolecule immobilization is a very vital step for a SMR sensor as it has dramatic effects on the selectivity and sensibility of sensor. The most popular biomolecule immobilization method is SAM as the cross-linker between biomolecules and the Au electrode surface. The SAM technique offers a simple way to form a fast, reproducible, ultrathin and well-ordered layer suitable for further modification. ${ }^{31,32}$ To start, the electrode surface was pretreated sequentially with piranha solution $\left(\mathrm{H}_{2}\right.$ $\mathrm{SO}_{4}: \mathrm{H}_{2} \mathrm{O}_{2}=3: 1$ ) for $5 \mathrm{~min}$, acetone for $5 \mathrm{~min}$ and ethanol for 5 min to obtain a clean and hydrophilic gold surface. Then the immobilized surface was washed repeatedly with distilled water and dried with pure $\mathrm{N}_{2}$. After that, the resonance frequency $f_{0}$ of the SAM was recorded as a reference. To continue the experiment, the electrode surface was covered with a monolayer of 11MUA by immersed in $1 \mathrm{mmol}^{-1}$ 11-MUA ethanolic solution for $24 \mathrm{~h}$ at room temperature. Then, the electrode surface was washed repeatedly with ethanol and distilled water in sequence and dried with pure $\mathrm{N}_{2}$. After that, $0.2 \mathrm{~mol}^{-1} \mathrm{EDC}$ and $0.05 \mathrm{~mol}$ $1^{-1}$ NHS solutions were mixed uniformity and applied onto the electrode surface for $1 \mathrm{~h}$ at room temperature to further activate the electrode surface. Then, $1 \mu \mathrm{l}$ of $0.5 \mathrm{mg} \mathrm{ml}^{-1}$ human IgG antibody was applied onto the surface of SMR for $2 \mathrm{~h}$ at $37^{\circ} \mathrm{C}$ to achieve complete binding between the human IgG antibody and the modified electrode surface. Again, the electrode surface was washed repeatedly with PBS and distilled water in sequence to remove physically adsorbed human IgG antibody and dried with pure $\mathrm{N}_{2}$. To complete the process, the electrode surface was immersed in $10 \mathrm{mg} \mathrm{ml}^{-1} \mathrm{BSA}$ solution for $2 \mathrm{~h}$ at $37^{\circ} \mathrm{C}$ subsequently to apply onto antibody-coated to block the unreacted sites and minimize unspecific effects. ${ }^{33}$ After that, a frequency measurement of SMR was carried out again and recorded as $f_{1}$.

For detecting the sensibility and selectivity of the SMR sensor, an amount of $1 \mu \mathrm{l}$ of $0.5 \mathrm{mg} \mathrm{ml}^{-1}$ goat anti-human IgG antigen with fluorescein isothiocyanate (FITC) was applied onto the electrode surface coated with human IgG antibody for $2 \mathrm{~h}$ at $37^{\circ} \mathrm{C}$ in the dark to achieve complete specific binding between the goat anti-human IgG antigen and human IgG antibody. Then, the electrode surface was washed repeatedly with PBS and distilled water in sequence to remove physically adsorbed goat anti-human IgG antigen and dried with pure $\mathrm{N}_{2}$. Finally, a frequency characteristic of SMR was measured again and recorded as $f_{2}$. The goat anti-human IgG antigen with FITC immobilization on the electrode surface was confirmed by fluorescence interference microscope (FIM) imaging. The schematic illustration of biomolecules immobilization on the electrode surface of SMR is shown in Fig. 2.

\section{Results and discussions}

Fig. 3 shows the XRD patterns of the SMR grown on silicon substrate. The diffraction peaks of silicon, Ti, W, and AlN can be observed clearly from the figure, confirming the structural integrity of SMR fabricated. Specially, for the AlN thin film, a very strong diffraction peak was observed at $35.94^{\circ}$ with a full width at half-maximum (FWHM) of $0.30^{\circ}$, which corresponds to 
Table 1 The specific sputtering parameters of Ti, W, AIN and Au

\begin{tabular}{lllll}
\hline Sputtering parameters & $\mathrm{Ti}$ & $\mathrm{W}$ & $\mathrm{AlN}$ & $\mathrm{Au}$ \\
\hline Target & $\mathrm{Ti}(99.999 \%)$ & $\mathrm{W}(99.999 \%)$ & $\mathrm{Al}(99.999 \%)$ & $\mathrm{Au}(99.999 \%)$ \\
RF power (W) & 150 & 150 & 250 & 150 \\
Ar flow rate (sccm) & 20 & 20 & 20 & 20 \\
$\mathrm{~N}_{2}$ flow rate (sccm) & 0 & 0 & 1.5 & 0 \\
Sputtering pressure (Pa) & 1.5 & 1.5 & 300 & 1.5 \\
Substrate temperature $\left({ }^{\circ} \mathrm{C}\right)$ & 25 & 25 & 2360 & 25 \\
Deposition thickness $(\mathrm{nm})$ & 630 & 570 & & 120
\end{tabular}
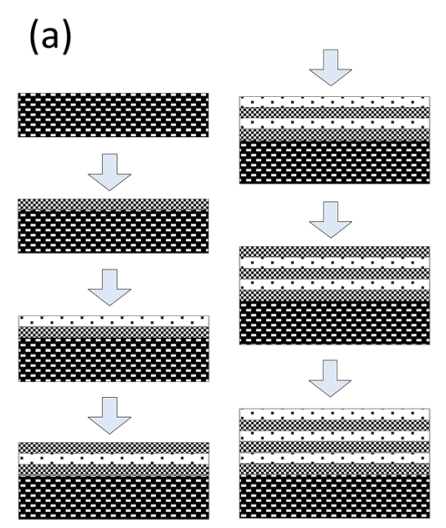

(b)

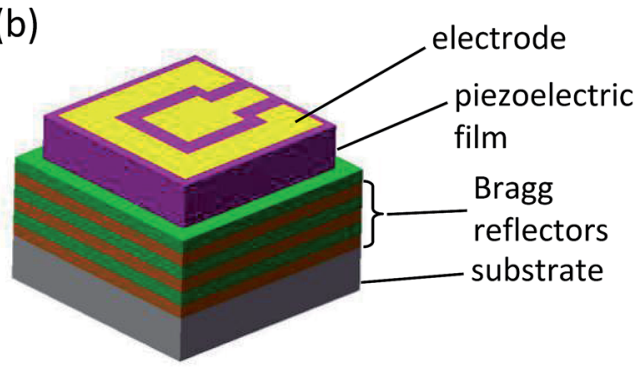

Fig. 1 The fabrication process flow and schematic illustration of SMR.

the diffraction from the AlN (002) plane. This verified that the preferential AlN growth orientation was along the wurtzite $c$-axis and perpendicular to the surface of substrate. In addition, according to the well-known Scherrer formula, ${ }^{34}$ the crystalline grain size of AlN was calculated to be about $28 \mathrm{~nm}$.

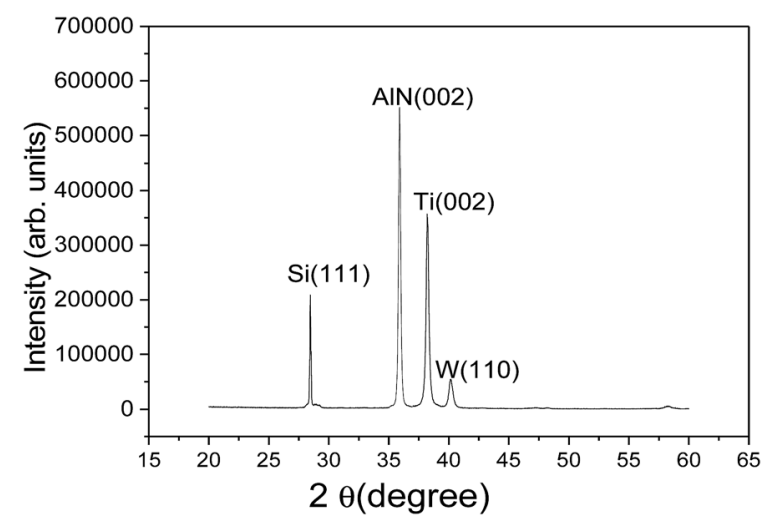

Fig. 3 The XRD patterns of SMR grown on silicon substrate.
Fig. 4 shows the cross-section view morphologies of the integrated SMR with $\mathrm{Au} / \mathrm{AlN} / \mathrm{Ti} / \mathrm{W}$ layered structure and the inset is the top electrode pattern. The AlN film perpendicular to the Bragg reflector exhibits highly oriented and compact columnar structure. The interfaces between the AlN film and Bragg reflector are clearly visible and distinct, verifying that the different membrane layers are not diffusive with each other. As the Bragg reflector was made entirely of metal, it had small internal stress and good heat conduction. Note that bottom Ti layer of the Bragg reflector on the top of Si also served as the electrode for frequency measurements.

The reflection coefficient $S(1,1)$, impedance and phase response of SMR were measured with probe station and network analyzer and displayed in Fig. 5, respectively. A distinct resonant phenomenon was observed clearly, indicated the good quality of the SMR we fabricated. The frequency response of SMR with $\mathrm{Ti} / \mathrm{W}$ Bragg reflector exhibited a return loss of $-20 \mathrm{~dB}$ at the center of $2.22 \mathrm{GHz}$. There were no other resonances owing to shear modes and higher-order harmonics from the picture, which verifies that the Bragg reflector had a good effect on successfully restraining other frequency resonant and stopping the acoustic dissipation to the substrate. ${ }^{35}$ The series and parallel resonant frequencies of SMR appeared at $2.20 \mathrm{GHz}$ and 2.23 $\mathrm{GHz}$, respectively. Both the $k_{\text {eff }}^{2}$ and the $Q$ of SMR can be derived easily by the expression from the $S$-parameter measurements as follows: ${ }^{36}$

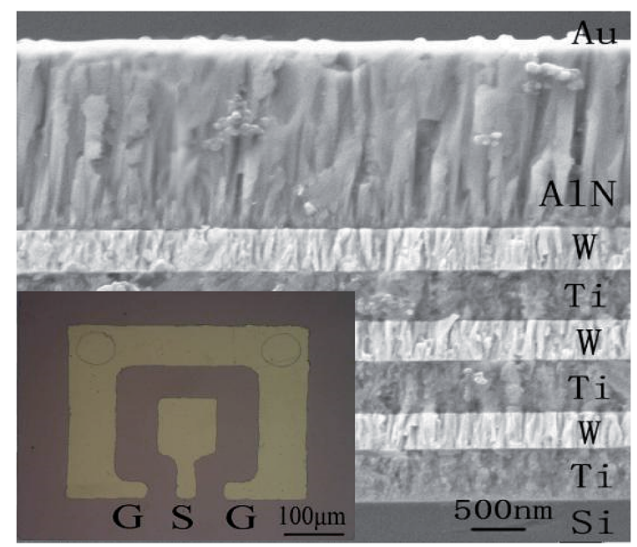

Fig. 4 The cross-section view morphologies of the integrated SMR. The inset is a top electrode pattern. 

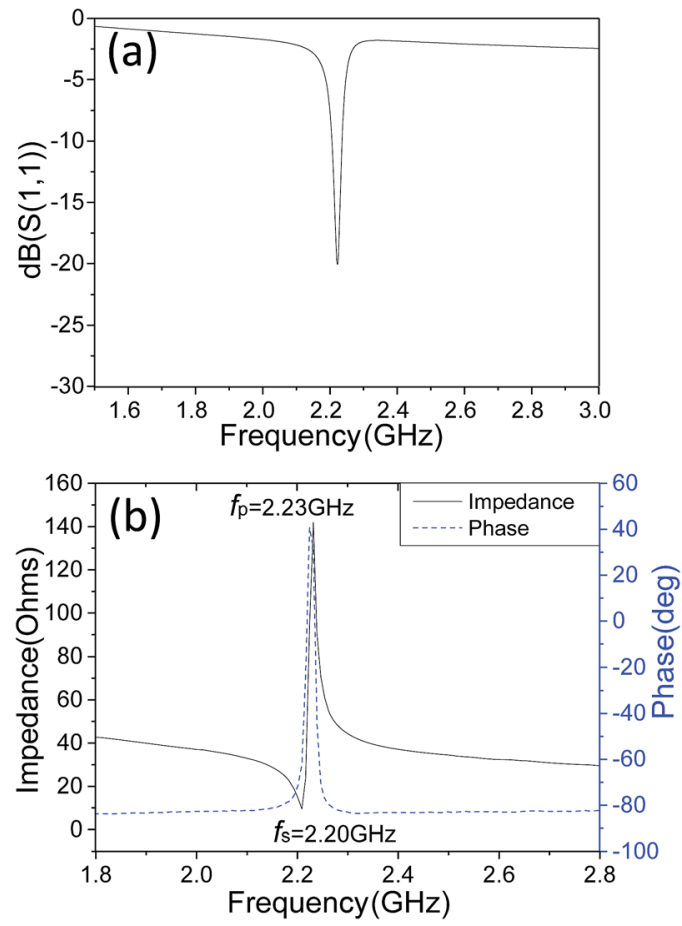

Fig. 5 The reflection coefficient $S(1,1)$, impedance and phase response of SMR.

$$
\begin{gathered}
k_{\mathrm{eff}}^{2}=\frac{\pi^{2}}{4}\left(\frac{f_{\mathrm{p}}-f_{\mathrm{s}}}{f_{\mathrm{p}}}\right) \\
Q_{\mathrm{s} / \mathrm{P}}=\frac{1}{2} f_{\mathrm{s} / \mathrm{p}}\left(\frac{\partial \angle Z}{\partial f}\right)_{\mathrm{s} / \mathrm{p}}
\end{gathered}
$$

where $f_{\mathrm{p}}$ and $f_{\mathrm{s}}$ are parallel and series frequencies of SMR, $Q_{\mathrm{S}}$ and $Q_{\mathrm{P}}$ are parallel and series quality factors and $Z$ is the input electrical impedance. According to calculation above, the $k_{\text {eff }}^{2}$, $Q_{\mathrm{S}}$ and $Q_{\mathrm{P}}$ of our SMR are $3.42 \%, 385$, and 505, respectively.

Fig. 6 shows the resonant frequency shifts of the SMR after biomolecules adsorption, due to the mass loading effect. Prior to the mass-loading, the resonance peak $f_{0}$ appeared at 2.220 GHz. The resonance peak $f_{1}$ drifted down $2.190 \mathrm{GHz}$ after

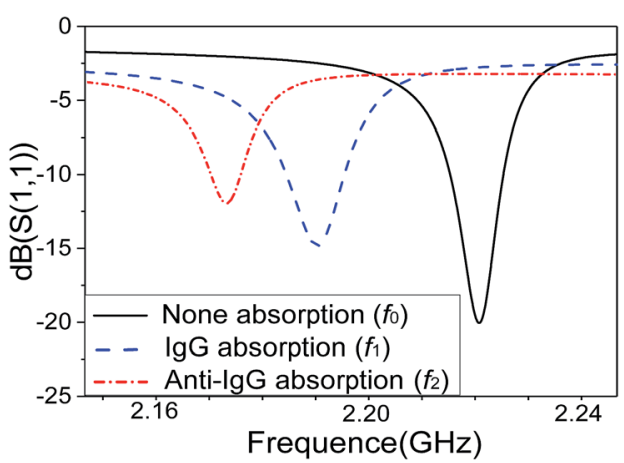

Fig. 6 The resonant frequency shifts of the SMR after biomolecules adsorption. absorbed human IgG antibody and BAS on the surface by the SAM method. Finally, the resonance peak $f_{2}$ drifted down 2.170 $\mathrm{GHz}$ after binding goat anti-human IgG antigen with FITC on the surface. The result demonstrates clearly that the resonance frequency drifted down significantly by absorbing a small amount of biomolecules onto the surface due to the massloading effect confirming that the SMR can be used effectively as a sensor for biomolecule detections.

The sensitivity of SMR can be estimated conveniently by Sauerbrey's formula as follow: ${ }^{37}$

$$
S_{\mathrm{m}}=\frac{2 f^{2}}{A \sqrt{\rho c}}
$$

where $S_{\mathrm{m}}$ is the mass sensitivity, $f$ is the unloaded resonance frequency, $\rho$ is the AlN mass density, $A$ is the area of operation and $c$ is a stiffness constant of the AlN piezoelectric material. According to eqn (3), the goat anti-human IgG antigen sensitivity of our SMR sensor is estimated to be $3.15 \mathrm{kHz} \mathrm{cm}^{2} \mathrm{ng}^{-1}$. The result demonstrates that our SMR sensor is highly promising in biomolecule detections because of its high sensitivity, small size, and low-cost than conventional QCM. ${ }^{38,39}$

In order verify the goat anti-human IgG antigen with FITC really immobilized on the Au electrode surface and caused a resonance frequency shift of SMR, the SMR sensor was observed by FIM and the micrograph was shown in Fig. 7. Nothing can be observed on the surface before the goat antihuman IgG antigen with FITC immobilized. After antigen immobilized, it is clearly observed that the top electrode was covered with fluorescent material that mainly consists of goat anti-human IgG antigen with FITC from the picture.

The relationship between the resonant frequency shift of human IgG antibody immobilized SMR sensor and the concentration of goat anti-human IgG antigen ranging from 0 to $0.7 \mathrm{mg} \mathrm{ml}^{-1}$ is shown in Fig. 8. There was a nearly linear relationship between the resonant frequency shift and the concentration of goat anti-human IgG antigen from 0 to $0.4 \mathrm{mg} \mathrm{ml} \mathrm{m}^{-1}$. However, the frequency shift was relatively constant when the concentration of antigen was greater than $0.5 \mathrm{mg} \mathrm{ml}^{-1}$, indicating the attainment of antibody adsorption plateau. When the concentration of antigen was too

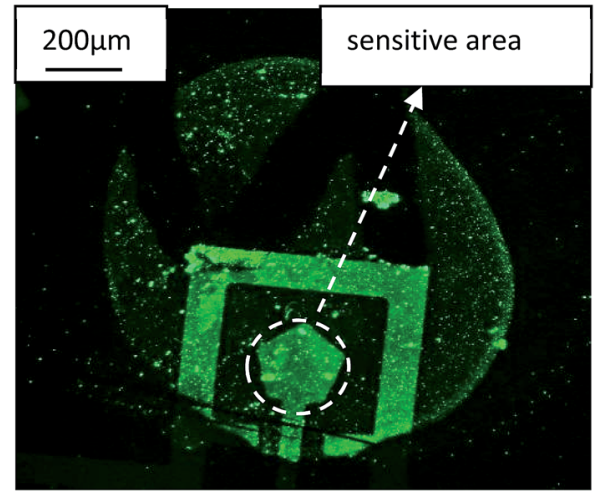

Fig. 7 The FIM micrograph of the goat anti-human IgG antigen with FITC immobilized on the SMR surface. 


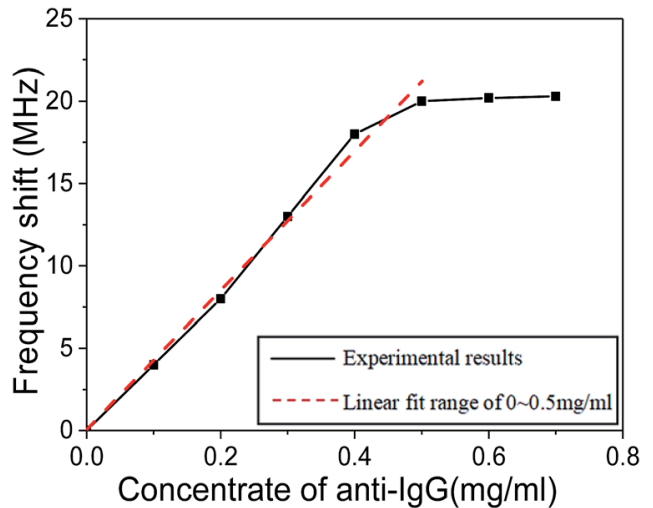

Fig. 8 The relationship between the resonant frequency shift of SMR sensor and the concentration of goat anti-human IgG antigen.

high, there was not enough human IgG antibody on the electrode surface for binding, which is the reason why the resonant frequency shift was not obvious. As a result, $0.5 \mathrm{mg}$ $\mathrm{ml}^{-1}$ of goat anti-human IgG antigen concentration was recommended as optimal concentration for SMR sensor.

In order to evaluate the specificity of SMR sensor, a series of interference experiments were also carried out. The human IgG antibody immobilized SMR was used to detect several endogenous substances such as human IgG, goat anti-mouse IgG, goat anti-rabbit IgG and BSA. The response frequency shift of SMR sensor to other endogenous substances were shown intuitively in Fig. 9. Adsorption goat anti-human IgG on human IgG antibody immobilized SMR surface resulted in a frequency shift down of $20 \mathrm{MHz}$, which demonstrates a higher sensitivity to target antigen. The introduction of human IgG, goat anti-mouse IgG, goat antirabbit IgG and BSA on human IgG antibody immobilized SMR surface only resulted in the frequency shift lower than 2 $\mathrm{MHz}$, which indicates the non-specific bindings were negligible. These results suggest that the SMR sensor was able to discriminate goat anti-human IgG from other endogenous substances easily and the selectivity of SMR sensor was satisfactory.

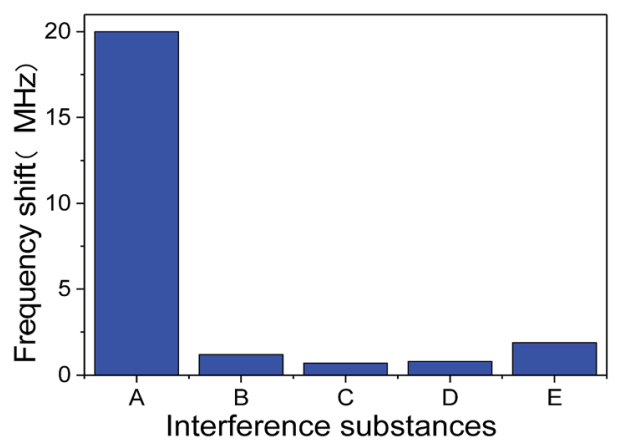

Fig. 9 The response frequency shift of SMR sensor to other endogenous interference substances at $0.5 \mathrm{mg} \mathrm{ml}^{-1}$ concentration. (A is goat anti-human $\lg G, B$ is human $\lg G, C$ is goat anti-mouse $\lg G, D$ is goat anti-rabbit $\lg G$ and $E$ is $B S A)$.

\section{Conclusions}

In this study, we have fabricated a SMR sensor for detection of goat anti-human IgG antigen based on the specific binding between goat anti-human IgG antigen and human IgG antibody. The SMR consisting of a thin AlN piezoelectric film and Bragg acoustic reflector with a resonant frequency of $2.22 \mathrm{GHz}$, and the $k_{\text {eff }}^{2}, Q_{\mathrm{S}}$ and $Q_{\mathrm{P}}$ of SMR are $3.42 \%, 385$ and 505, respectively. Human IgG antibody was immobilized on the electrode surface of SMR through SAM method for future detection. Then, goat anti-human IgG antigen with FITC was immobilized on the electrode surface of SMR through the specificity of bind between antibody and antigen and can be observed by FIM, clearly. The sensitivity of the SMR sensor for goat anti-human IgG antigen was estimated to be $3.15 \mathrm{kHz} \mathrm{cm}^{2} \mathrm{ng}^{-1}$. The relationship between the resonant frequency shifts of SMR sensor and the concentrations of goat anti-human IgG antigen ranging from 0 to $0.7 \mathrm{mg} \mathrm{ml}^{-1}$ was investigated and $0.5 \mathrm{mg} \mathrm{ml}^{-1}$ was recommended as optimal concentration. A series of interference experiments were also applied to evaluate the specificity of SMR sensor and the result suggested the selectivity of sensor was satisfactory. Lastly, our SMR sensor provides a new approach for detection of goat anti-human IgG antigen. It also shows a promising future for biomolecule detections in medical diagnostics and environmental protections.

\section{Conflicts of interest}

There are no conflicts to declare.

\section{Acknowledgements}

This work is supported by the Key Research and Development Program of Shandong Province (2018GGX101008) and National Natural Science Foundation of China (11874232).

\section{Notes and references}

1 X. Duan, Y. Li, N. K. Rajan, D. A. Routenberg, Y. Modis and M. A. Reed, Nat. Nanotechnol., 2012, 7(6), 401-407.

2 L. Mu, I. A. Droujinine, N. K. Rajan, S. D. Sawtelleet and M. A. Reed, Nano Lett., 2014, 14(9), 5315-5322.

3 P. M. Kosaka, V. Pini, J. J. Ruz, R. A. da Silva, M. UjueGonzález, D. Ramos, M. Calleja and J. Tamayo, Nat. Nanotechnol., 2014, 9(12), 1047-1053.

4 S. Scarano, C. Scuffi, M. Mascini and M. Minunni, Biosens. Bioelectron., 2010, 25(5), 957-966.

5 W. Guan, N. K. Rajan, X. Duan and M. A. Reed, Lab Chip, 2013, 13(7), 1431-1436.

6 A. Ramachandran, S. Wang, J. Clarke, S. J. Ja, D. Goad, L. Wald, et al., Biosens. Bioelectron., 2008, 23(7), 939-944.

7 Z. Altintas, Y. Uludag, Y. Gurbuz and I. Tothill, Anal. Chim. Acta, 2012, 712, 138-144.

8 N. B. Muren and J. K. Barton, J. Am. Chem. Soc., 2013, 135(44), 16632-16640.

9 I. Voiculescu and A. N. Nordin, Biosens. Bioelectron., 2012, 33(1), 1-9. 
10 Q. Yang, S. Pan, Y. Zhao, H. Zhang, W. Pang and X. Duan, Biosens. Bioelectron., 2017, 96, 206-212.

11 X. Zhao, F. Pan, G. M. Ashley, L. Garcia-Gancedo, J. Luo, A. J. Flewitt, et al., Sens. Actuators, B, 2014, 190(1), 946-953.

12 T. Wang, X. Mu, A. B. Randles and Y. D. Gu, Appl. Phys. Lett., 2015, $107(12), 12350$.

13 J. G. RodríguezMadrid, G. F. Iriarte, O. A. Williams and F. Calle, Sens. Actuators, A, 2013, 189(2), 364-369.

14 D. D. Erbahar, I. Gurol, G. Gümüş, E. Musluoğlu, Z. Z. Öztürk, V. Ahsen and M. Harbeck, Sens. Actuators, B, 2012, 173, 562-568.

15 C. Cheng, Y. Chang and Y. Chu, Chem. Soc. Rev., 2012, 41(5), 1947-1971.

16 G. S. Gill and M. Prasad, Sens. Lett., 2016, 14(4), 346-361.

17 M. L. Johnston, I. Kymissis and K. L. Shepard, IEEE Sens. J., 2010, 10(6), 1042-1047.

18 N. M. Torres, Sensors, 2010, 10(5), 4180-4193.

19 H. Guo, Y. Gao and T. Liu, Measurement, 2018, 129, 206-210.

20 L. Fan, S. Zhang, H. Ge and H. Zhang, J. Appl. Phys., 2013, $114(2), 0245$.

21 G. Piazza, P. J. Stephanou and A. P. Pisano, J. Microelectromech. Syst., 2006, 15(6), 1406-1418.

22 P. Guo, J. Xiong, D. Zheng, W. Zhang, L. Liu, S. Wang and H. Gu, RSC Adv., 2015, 5(81), 66355-66359.

23 X. He, L. Garcia-Gancedo, P. Jin, J. Zhou, W. Wang, S. Dong, et al., J. Micromech. Microeng., 2012, 22(22), 1519-1529.

24 L. M. Clement, A. Delicado, R. J. Olivares, T. Mirea, J. Sangrador and E. Iborra, Sens. Actuators, A, 2017, 258, 39-43.

25 C. Wei, Y. Chen, S. Li, C. Cheng, K. Kao and C. Chung, Appl. Phys. A, 2010, 99(1), 271-278.
26 M. Knapp, R. Hoffmann, V. Lebedev, V. Cimalla and O. Ambacher, Nanotechnology, 2018, 29(10), 1-10.

27 Y. Satoh, T. Nishihara, T. Yokoyama, M. Ueda and T. Miyashita, Jpn. J. Appl. Phys., 2005, 44(5A), 2883-2894.

28 G. Rughoobur, H. Sugime, M. Demiguel-Ramos, T. Mirea, S. Zheng, J. Robertson, et al., Sens. Actuators, B, 2018, 261, 398-407.

29 W. Pang, H. Zhao, E. S. Kim, H. Zhang, H. Yu and X. Hu, Lab Chip, 2012, 12(1), 29-44.

30 C. Han, D. Chen, Y. Zhang, D. Xu, Y. Liu, E. Kong, et al., Nano-Micro Lett., 2012, 4, 40-44.

31 S. Carrara, L. Benini, V. Bhalla, C. Stagni, A. Ferretti, A. Cavallini, et al., Biosens. Bioelectron., 2009, 24(12), 34253429.

32 W. Tsai and I. Lin, Sens. Actuators, B, 2005, 106(1), 455-460. 33 A. Tang, M. Pravda, G. Guilbault, S. Piletsky and A. Turner, Anal. Chim. Acta, 2002, 471(1), 33-40.

34 S. Lin, J. Huang and D. Lii, Mater. Chem. Phys., 2005, 90(1), 22-30.

35 M. DeMiguel-Ramos, B. Díaz-Durán, J. Escolano, M. Barba, T. Mirea, J. Olivares, et al., Sens. Actuators, B, 2017, 239, 1282-1288.

36 Q. Su, R. Kirby, E. Komuro, M. Imura, Q. Zhang and R. Whatmore, IEEE Trans. Microwave Theory Tech., 2001, 49(4), 769-778.

37 G. Sauerbrey, Z. Phys., 1959, 155(2), 206-222.

38 G. Wingqvist and H. Anderson, Biosens. Bioelectron., 2009, 24(11), 3387-3390.

39 Y. Q. Fu, J. K. Luo, X. Y. Du, A. J. Flewitt, Y. Li, G. H. Markx, et al., Sens. Actuators, B, 2010, 143(2), 606-619. 